\title{
Acute Management of an Abductor Digiti Minimi Strain in a Collegiate Baseball Player: A Patient-Centered Case Study
}

S. Andrew Cage, MEd, LAT, ATC1,2, Diana M. Gallegos, MS, LAT, ATC', Brandon J. Warner, MEd, LAT, ATC2,3

'The University of Texas at Tyler, Tyler, TX; 2The University of North Carolina Greensboro, Greensboro, NC, ${ }^{3}$ Grand Canyon University, Phoenix, AZ

\begin{abstract}
The purpose of this disablement model case study was to describe the case of a collegiate baseball player suffering from an isolated abductor digiti minimi strain. Despite an initial decrease in function, the treating sports medicine staff was able to provide the necessary care to allow the patient to participate in the final games of his career. While the anatomy and function of abductor digiti minimi are well described in the literature, there is no documentation of isolated abductor digiti minimi injuries. In this case, the patient injured the medial aspect of his hand when hitting a baseball. The patient experienced immediate pain along with the sensation of "tightening" throughout the hypothenar eminence. The following day the patient reported difficulty with sleeping and daily activities due to pain. Although fracture tests were negative, the patient exhibited significant weakness and pain with fifth finger abduction and flexion leading to physician referral. The team physician noted pain with active ulnar deviation in addition to previous symptoms. At this time, the patient was diagnosed with an abductor digiti minimi strain. The patient stated that he was in his final season of competition and wished to continue participating with his team. The patient consented to a treatment plan involving local injection of lidocaine and bupivacaine along with compression via Kinesiology Tape $\AA$. Using these methods, the patient was able to participate in the final two games of his career. Following the cessation of baseball activities, the patient noted that he had no recurrent symptoms or complications from his injury. When prescribing treatment for patients in a competitive setting, it is paramount that clinicians take into account patient centered values. If there is no risk of significant, long term injury, clinicians should attempt to provide patients with the means to participate in activities if they should desire.
\end{abstract}

\section{Key Phrases}

Abductor digiti minimi, college and university patient population, functional testing

\section{Correspondence}

S. Andrew Cage, The University of Texas Tyler, 11325

Preakness Dr., Flint, TX 75762.

E-mail: sacage@uncg.edu

\section{Full Citation}

Cage SA, Gallegos DM, Warner BJ. Acute Management of an Abductor Digiti Minimi Strain in a Collegiate Baseball Player: A Case Study. Clin Pract Athl Train. 2018;1(2):9-13. https://doi.org/10.31622/2018/0002.3.

Submitted: August 16, 2018 Accepted: October 1, 2018

\section{INTRODUCTION}

The abductor digiti minimi is a small muscle that makes up a portion of the hypothenar eminence. Originating from the pisiform, pisohamate ligament, and flexor retinaculum, the abductor inserts on the proximal phalanx of the fifth digit as well as the sesamoid bone when present. Primarily, this muscle is responsible for the abduction of the fifth digit. ${ }^{1}$ The muscle receives its neurological innervation from the deep branch of the ulnar nerve and vascular supply from the ulnar artery. ${ }^{2}$ Apart from its role in fifth digit abduction, no other functions have been described for the abductor digiti minimi.

The abductor digiti minimi has been implicated in approximately a quarter of the cases of Dupuytren's contracture of the hand. ${ }^{3}$ In some cases, this compression of the ulnar nerve can be caused by the presence of an accessory abductor digiti minimi muscle. ${ }^{4}$ In spite of the abductor digiti minimi's involvement in ulnar nerve pathologies, there is no peer reviewed literature describing an isolated injury to the abductor digiti minimi. 3,4 Furthermore, without a body of literature, it is difficult to create an evidence based approach to treating these injuries and the subsequent dysfunctions that occur. Thus, it is the purpose of this case study to describe an isolated abductor digiti minimi in a collegiate baseball player. This case will describe the mechanism of injury, 
diagnosis, treatment, patient reported disablements, and outcomes.

\section{Patient Information}

The patient described in this case is a right 22year-old collegiate baseball player who fielded the shortstop position. When playing, the patient would throw right-handed and bat left-handed. The patient had a previous history of surgical excision of the hook of the hamate in his right hand from the previous year, but was otherwise healthy and fully functional. After hitting a baseball during competition, the patient reported immediate pain along the medial aspect of his right hand. Initial evaluation revealed moderate pain, but full strength and range of motion with all wrist and digit motions. For the remainder of the game, the patient was able to participate at full capacity, but was instructed to report to the athletic training staff the following day.

\section{Differential Diagnosis and Evaluation}

The following day, the patient reported to the athletic training clinic complaining of increased pain. The patient stated that the pain had disrupted his sleep, made it difficult to turn the steering wheel of his car while driving to the clinic, and was significantly worse than the previous day. The patient's hand was diffusely tender to palpation along the medial aspect of the hypothenar eminence. While all fracture tests were negative, the patient presented with enough pain and weakness with fifth digit abduction and grip strength that physician referral was warranted. At this time, the differential diagnosis included: TFCC injury, Wrist Sprain, Wrist Strain, Subluxing Extensor Carpi Ulnaris.

Two days after the initial injury during the team physician's evaluation, it was also noted that the patient was experiencing pain with ulnar deviation. However, the most explicit pain and weakness was elicited with resisted fifth digit

Copyright (C) by Indiana State University All rights reserved. ISSN Online 2577-8188 abduction. Following a comprehensive exam, the patient was diagnosed with an abductor digiti minimi strain. It was at this time that the patient expressed his concern regarding his ability to participate in the upcoming tournament. His concern were compounded by the fact that he was in his last year of eligibility, and the upcoming tournament represented his last opportunity to participate in collegiate baseball.

With the patient's desires and values in mind, treatment options were discussed, and the patient was educated on the potential outcomes of all of them. Given that other conservative treatment options would have resulted in a significant delay in returning to competition, the decision was ultimately made to perform local injections of lidocaine and bupivacaine at the insertion of the abductor digiti minimi prior to the upcoming competitions in addition to being taped to provide as much compression as possible without inhibiting function (Figure 1). Kinesiology Tape $\mathbb{R}$ was chosen to allow the patient the most range of motion possible at the wrist while also providing compression. A first strip of tape was cut and then applied over the medial aspect of the hypothenar eminence with $50 \%$ tension. A second strip of tape was split half way, with the anchor covering the ulnar styloid. Once the anchor was affixed, the split ends of the tape were then wrapped around the wrist medial to lateral in an effort to provide as much compression as possible without occluding neurovascular structures.

\section{Body Structure and Function}

Given that the injury was muscular in nature, the primary diagnostic tools utilized were strength and range of motion tests. At the initial time of injury, the patient presented with full range of motion and adequate wrist and digit strength with only mild pain. However, by the following day, the patient's strength with fifth digit abduction had decreased to a $4 / 5$. The patient was still able to perform ulnar deviation with full 


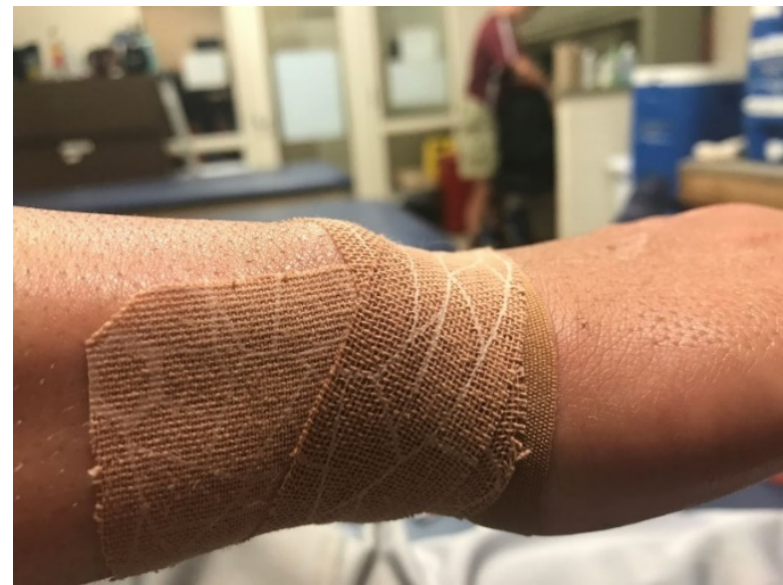

Figure 1: Compression tape for digiti mini strain.

strength, but noted increased pain from the previous day. Additionally, the patient could no longer perform the motion without experiencing significant pain.

\section{Activity and Participation}

In order to determine if the patient would be able to participate in the upcoming games, functional testing was performed consisting of hitting and throwing drills prior to the initiation of interventions. With the decrease in strength and increase in pain, the patient began to experience difficulty gripping his bat and throwing with both accuracy and velocity. If the patient decreased his velocity he could throw more accurately, but he expressed concern that this may affect his ability to remain competitive at his position. The patient stated that he believed that he would be able to improve both the accuracy and velocity of his throws if his pain levels were decreased.

\section{Environmental and Personal Factors}

Outside of baseball related activities, the patient stated that the pain he was experiencing in his hand was affecting his daily activities.

Specifically, the patient stated that the intensity of pain inhibited his ability to obtain quality sleep and drive his motor vehicle. Other activities that the patient's hand pain affected included being able to type on his computer. The patient

Copyright (C) by Indiana State University All rights reserved. ISSN Online 2577-8188 reported having to change his typing form in order to mitigate the intensity of pain he was experiencing. This change in form resulted in the patient's homework assignment taking significantly longer than it would have otherwise. Given the inconveniences created for the patient's in his collegiate activities and in his daily life, he wished for his injury to be healed as quickly as possible

\section{INTERVENTIONS}

Three days post injury, the patient was seen in the athletic training room prior to his first postseason competition. The patient's skin was prepped using an alcohol prep pad, and a mixture of lidocaine and bupivacaine was injected near the proximal aspect of the hypothenar eminence. Two strips of Kinesiology Tape ${ }^{\circledR}$ were then used to provide as much compression as possible without impeding wrist or hand motion. While this technique had not necessarily been described in the current literature, the clinician used knowledge of the structures being taped and the principles behind compression tape to approximate the best tape job possible for the goals in mind. Follow up evaluation revealed that the patient did not experience any further pain at rest, or with motion, and demonstrated full strength with fifth digit abduction and grip strength. The patient was then taken to the field to ensure that he would be able to grip and swing a bat, and grip and throw a baseball. After demonstrating the ability to swing a bat without pain, the patient then hit off of a tee to provide resistance to his swing. Once the patient had passed this sportspecific testing, the patient was cleared to participate in the day's competition.

\section{OUTCOMES}

\section{Body Structure and Function}

The patient was able to regain fifth finger abduction, wrist ulnar deviation, and grip 
strength after the application of lidocaine and bupivacaine injections in combination with compression taping at the proximal aspect of the hypothenar eminence. Along with the recovered strength, the patient noted that there was little to no pain for roughly three hours post injection. However, after the three hour time frame had elapsed, his pain gradually increased again. When asked, the patient said this period of relatively pain-free activity was enough to allow him to comfortably and effectively participate in his competitions at full function.

\section{Activity and Participation}

Through the use of the proposed treatment plan, the patient was able to participate in the final two contests of his career. While participating, the patient was able to perform at a high level and maintain the standard of performance he had throughout the season. The patient noted that if any of the competitions had gone longer than their regulation length, he may have had increased difficulty in completing in them, but had no severe issues completing a standard game.

Aside from baseball, both sleeping and activities of daily living that required gripping or fifth digit abduction continued to bother him. If the injury had taken place during the regular season, the patient may have required considerations from his instructors regarding completion of course work that required a large amount of typing or writing. Fortunately, the academic year had concluded by this point in the season, and the patient was not required to perform extensive writing or typing that might have exacerbated the symptoms of his injury. Within a week of completing his final season, the patient reported significant decreases in pain following relative rest combined with regular icing and NSAID usage, and was pain free by the twoweek mark.

\section{Environmental and Personal Factors}

Copyright ( $)$ by Indiana State University All rights reserved. ISSN Online 2577-8188
Given the patient's expressed desire to participate in the final competitions of his playing career, he remained stringently adherent to all appointments and measures taken to allow him to participate. While the patient reported a slight increase in pain from baseline following each of the two competitions, he stated that is was well within tolerable limits if it meant he was allowed to continue to play. Furthermore, the patient was not allergic to lidocaine, bupivacaine, or the adhesive from the Kinesiology Tape ${ }^{\circledR}$. As such, the patient suffered from no adverse effects from the chosen treatment course.

\section{DISCUSSION}

This case describes the diagnosis and management of a patient suffering from an isolated abductor digiti minimi strain. While the abductor digiti minimi's involvement in other pathologies has been described, there is no documentation detailing strains to the muscle and the treatment thereof. 3,4 Fortunately, this case resulted in a positive outcome for the patient which allowed him to achieve his goals. However, the short timeframe did not allow for a measurement of progression of the injury while continuing participation in baseball. Had this injury occurred at a different phase of the season, the clinician would have been able to attempt other conservative treatments and chart the progression of the patient's outcomes in order to determine the best possible course of treatment in future instances. This means that the case presented may not be generalizable for clinicians seeking to care for a patient with a similar injury over a longer course of time.

Further research is required to determine the best practices for managing an acutely strained abductor digiti minimi in a patient who requires above average dexterity to complete their daily activities.

Overall, the choice of intervention (i.e. local anesthetic injection and compression taping) was 
chosen due to the short timeframe in which the patient had to recover from his injury. Had the patient had prospects of playing past the current season, there may have been other considerations when determining the course of treatment. Additionally, if the patient had been required to continue typing for his academic work there may have been a need for other interventions, including discussions with instructors of alternate methods for the patient to complete his assignments. Ultimately, the patient's symptoms were able to resolve with cessation of activity, and no adverse outcomes were reported.

\section{CLINICAL BOTTOM LINE}

Within the scope of clinical practice, it is entirely possible for clinicians to encounter injuries and conditions that are not well described in the literature. In these instances, clinicians must rely on their expertise along with patient-reported measures and values. When relying on these facets of clinical care, evaluation and reevaluation of the clinician's treatment and rehabilitation plan are crucial to achieving optimal patient outcomes. When prescribing a course of treatment for patients in a competitive setting, it is paramount that clinicians take into account patient-centered values. While the goal of clinicians should be to provide patientcentered care, they must also incorporate patient education as much as possible. By providing a patient with as much information as possible regarding potential outcomes of a chosen treatment program, the clinician can hopefully mitigate at least some of the potential conflicts of interest that may occur in such a scenario. If there is no risk of significant, long-term injury, clinicians should attempt to provide patients with the means to participate in activities if they should desire.

\section{REFERENCES:}

1. Schmidt UL. Surgical Anatomy of the Hand. 1 st ed. New York, NY: Thieme Medical Publishers, Inc; 2003.

2. Usyal AC, Alagoz MS, Tuccar E, Sensoz O, Tekdemir I. The vascular anatomy of the abductor digiti minimi and the flexor digitorum brevis muscles. J Hand Surg Am. 2005;30(1):172-176. https://doi.org/10.1016/i.jhsa.2004.06.00 1

3. Meathrel KE, Thoma A. Abductor digiti minimi involvement in Dupuytren's contracture of the small finger. J Hand Surg Am.

2004;29(3):510-513. https://doi.org/10.1016/i.jhsa.2004.01.01 $\underline{6}$

4. Al-Qattan MM. Ulnar nerve compression at the wrist by the accessory abductor digiti minimi muscle: wrist trauma as a precipitating factor. J Hand Surg. 2004;9(1):79-82. https://doi.org/10.1142/S021881040400 1899 